\title{
Influence of fatty deposits in the levator aponeurosis/levator palpebrae superioris muscle on outcomes of aponeurotic repair in a Japanese population
}

\author{
Hiromichi Matsuda ${ }^{1} \cdot$ Tsutomu Sakai $^{1} \cdot$ Yasuhiro Takahashi $^{2} \cdot$ Masaki Nakamura $^{3} \cdot$ Tadashi Nakano $^{3}$
}

Received: 18 February 2018 / Revised: 19 June 2018 / Accepted: 9 July 2018 / Published online: 16 August 2018

(c) The Royal College of Ophthalmologists 2018

\begin{abstract}
Purpose To examine a part of the levator aponeurosis/levator palpebrae superioris (LPS) muscle that did not have fatty deposits in patients with aponeurotic blepharoptosis. We also analysed the relation between this length and surgical outcome after transcutaneous aponeurotic repair.

Methods We measured the vertical length from the distal end of the anterior layer of the levator aponeurosis to the most distal point of fatty deposits in the levator aponeurosis/LPS muscle (non-fatty-deposit length) in 94 eyelids. Surgical success was defined as a postoperative margin reflex distance-1 (MRD-1) of 2.0-5.0 mm at 3 months postoperatively. The eyelids were classified into two groups: a surgical success group (group A, 76 eyelids) and an undercorrected group (group B, 18 eyelids). Group A was subdivided according to the evidence of a fatty deposit (group A1, fat present, 70 eyelids; group A2, fat absent, 6 eyelids).

Results The non-fatty-deposit measurement was significantly longer in group A1 than in group B $(p=0.035)$. The levator aponeurosis was less advanced in groups A1 and A2 than in group B (both, $p<0.001$ ), and the eyelids in groups A1 and A2 obtained a higher MRD-1 than those in group B (both, $p<0.001$ ).

Conclusions Eyelids with less fat deposit required less advancement of the levator aponeurosis and obtained more favourable surgical results. Intraoperative findings of eyelids with and without fatty deposits and measuring the non-fattydeposit length may be useful predictive parameters for outcomes of transcutaneous aponeurotic repair.
\end{abstract}

\section{Introduction}

Fatty deposition in the levator aponeurosis/levator palpebrae superioris (LPS) muscle is a cause of aponeurotic blepharoptosis $[1,2]$. Fatty degeneration predominantly occurs in the LPS muscle but occasionally extends to the levator aponeurosis [2]. Increased fatty deposition in the levator aponeurosis/LPS muscle causes weakness of the tractional force to the upper eyelid [2]. A previous study showed that

Hiromichi Matsuda

thkmmm@yahoo.co.jp

1 Department of Ophthalmology, Daisan Hospital, The Jikei University School of Medicine, Komae, Tokyo, Japan

2 Department of Oculoplastic, Orbital and Lacrimal Surgery, Aichi Medical University Hospital, Nagakute, Aichi, Japan

3 Department of Ophthalmology, The Jikei University School of Medicine, Tokyo, Japan a fatty deposit in the aponeurosis/LPS muscle is more prevalent in East Asians than in Caucasians [3], suggesting that fatty degeneration has a greater impact on the clinical aspects of aponeurotic blepharoptosis in East Asian patients. As far as we can determine, however, the influence of fatty degeneration on surgical outcomes of transcutaneous aponeurotic repair has not been explored in this population.

The levator aponeurosis is composed of anterior and posterior layers [4]. The anterior layer of the levator aponeurosis (ALLA) is a thick, robust fibrous tissue that reflects superiorly a few millimetres above the tarsal plate and continues to the orbital septum [4]. The posterior layer, composed of thinner fibrous tissue, attaches to the tarsal plate and extends to subcutaneous tissue [4, 5]. The distal end of the ALLA, called the "white line," is easily identifiable after incision of the orbital septum [6], and is often used as a reference point to determine the amount of advancement of the levator aponeurosis during blepharoptosis surgery [7]. We, therefore, chose to use the white 
line to estimate the degree of fatty degeneration in the levator aponeurosis/LPS muscle.

We evaluated the vertical distance from the distal end of the ALLA to the most distal point of the fatty deposit, indicating the area without a fatty deposit (non-fatty-deposit length), during transcutaneous aponeurotic repair and then analysed the relation between that length and surgical outcomes.

\section{Patients and methods}

This retrospective chart review included all patients who underwent transcutaneous aponeurotic repair for aponeurotic blepharoptosis performed by one oculoplastic surgeon (H.M.) between April 2014 and March 2017. Exclusion criteria included levator function of $<5 \mathrm{~mm}$, a follow-up period of $<3$ months, a history of upper eyelid surgery, or a lack of clinical data.

Institutional review board (IRB) approval was obtained from The Jikei Medical University (number 29-184), and the protocol adhered to the tenets of the Declaration of Helsinki. As it was not an interventional study, the IRB granted a waiver for informed consent for this study based on the ethical guidelines for medical and health research involving human subjects established by the Japanese Ministry of Education, Culture, Sports, Science, and Technology and by the Ministry of Health, Labour, and Welfare. Nevertheless, the IRB requested that we present an outlined description of this study to the public via a notice board in our institution to provide an additional opportunity for patients to refuse participation in this study. None of the patients declined to participate. Personal identifiers were removed from the records prior to data analysis.

The following data were collected from patients' medical records, facial photographs, and intraoperative videos: age, sex, surgical side, use of contact lenses, margin reflex distance (MRD)-1, levator function, response to phenylephrine eye drops, eyelid crease condition (including no, or a high, eyelid crease), presence or absence of fatty degeneration in the levator aponeurosis/LPS muscle, dehiscence or disinsertion of the levator aponeurosis, the non-fat-deposit length and postoperative complications. All preoperative and postoperative measurements (in units of $0.5 \mathrm{~mm}$ ) were performed using a millimetre ruler by two independent examiners (H.M. and M.N.) who had received the same instruction in oculoplasty. The non-fatty-deposit length was determined by the same examiners using an intraoperative video. The examiners did not know the results of the other investigator. When their measurement values were different, average values were used [8].

MRD-1 was measured before and 3 months after surgery. A 5\% phenylephrine test was performed preoperatively on

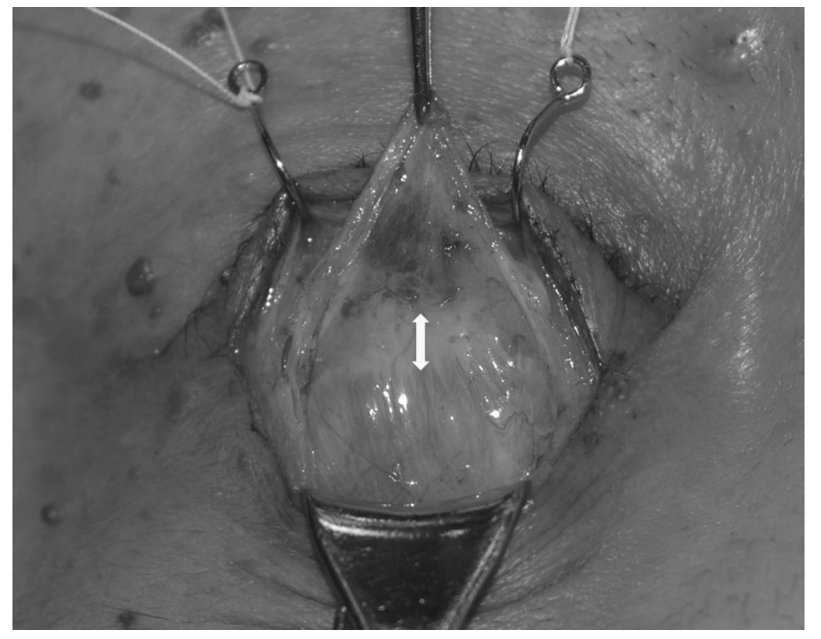

Fig. 1 Measuring the length from the distal end of the anterior layer of the levator aponeurosis to the most distal point of the fatty deposit. The measurement was carried out from the centre of the white line (arrow) (lower, cranial; right, temporal)

the surgical side [9]. Any changes in MRD-1 were recorded 20 min after application of the phenylephrine eye drops. A high eyelid crease was diagnosed when the crease height, measured from the upper eyelid margin during eyelid closure, was $\geq 7 \mathrm{~mm}[10]$. A fatty deposit in the LPS muscle \pm the levator aponeurosis was deemed positive in the presence of gross yellow tissue, compared with the pinkish to brownish muscle and white-glistening levator aponeurosis up to the level of several millimetres above the Whitnall ligament [11]. The non-fatty-deposit length was measured at the centre of the white line while pulling it inferiorly using forceps (Fig. 1). In patients without a fatty deposit in the levator aponeurosis/LPS muscle, the length of the ALLA was measured. Dehiscence or disinsertion of the levator aponeurosis was noted when the Müller muscle-a translucent, reddish layer of muscle fibres and blood vessels -was clearly observed between the attachment site of the orbital septum and the upper edge of the tarsal plate [11]. The amount of advancement of the levator aponeurosis was measured from the centre of the white line.

Surgical success was defined when each eyelid achieved a postoperative MRD-1 of $2.0-5.0 \mathrm{~mm}$. The eyelids were classified into two groups: group A (surgical success) and group B (undercorrected). Group A was then subdivided according to the evidence (or lack thereof) of a fatty deposit in the levator aponeurosis/LPS muscle (group A1, fat present; group A2, fat absent). All eyelids in group B were found to have a fatty deposit. The upper eyelid was not overcorrected (MRD-1 $\geq 5.5 \mathrm{~mm}$ ) in any patient 3 months postoperatively.

The patients' ages and the measurements were expressed as means \pm standard deviation. Intergroup differences in levator function, preoperative and postoperative MRD-1, 
response to phenylephrine eye drops and the amount of advancement of the levator aponeurosis were examined using a one-way analysis of variance and the Tukey-Kramer post hoc test. The surgical side ratios and the percentages of the eyelids with a double eyelid crease, high eyelid crease and dehiscence or disinsertion were compared between the groups using a $\chi^{2}$ test for independent variables. The non-fatty-deposit length was compared between groups A1 and B using the Mann-Whitney $U$ test. Logistic regression analysis was carried out to determine factors influencing surgical success. For the logistic regression analysis, all categorised factors were expressed using a binary system (a dummy variable, 0 and 1). Statistical analyses were performed using JMP version 13 software (SAS, Cary, NC, USA). A value of $p<0.05$ was considered to indicate statistical significance.

\section{Surgical technique}

The details of our surgical techniques were previously reported [12]. Briefly, marking for a 20- to 22-mm skin incision was made $7 \mathrm{~mm}$ above the eyelid margin. Local anaesthetic (1\% buffered lidocaine and epinephrine $1: 100,000)$ was injected subcutaneously around the skin incision line. After the skin incision, the layer under the orbicularis oculi muscle was dissected to expose the tarsal plate. In patients without dehiscence or disinsertion of the levator aponeurosis, the posterior layer of the levator aponeurosis was detached from the tarsal plate until insertion of the Müller muscle on the upper edge of the tarsal plate was exposed. The levator aponeurosis was bluntly dissected away from the Müller muscle. The orbital septum near the white line was incised transversely, and the ALLA was exposed [5]. The levator aponeurosis was advanced and secured to the upper one-third of the tarsal plate with 6-0 polyvinylidene fluoride sutures (Asflex ${ }^{\circledR}$; Kono Seisakusho, Tokyo, Japan). The advancement was repeated until an adequate eyelid height was obtained. When necessary, the levator aponeurosis was fixed to the tarsal plate at one or two more points to create a natural curvature. Finally, an eyelid crease was created at three points using 6-0 Asflex buried sutures, and the wound was closed with 6-0 Asflex sutures.

Postoperative medications consisted of oral levofloxacin for 3 days and $0.3 \%$ ofloxacin ointment and $0.5 \%$ topical levofloxacin for 2 weeks.

\section{Results}

The patients' demographic data, measurement results, and statistical comparisons between the groups are shown in Table 1. Among the 88 patients who underwent blepharoptosis surgery, 35 were excluded because of poor levator function $(n=7)$, insufficient follow-up period $(n=$ $3)$, prior blepharoptosis surgery $(n=6)$ or lack of data $(n=$ 19). Finally, the study included 94 eyelids (48 right and 46 left) of 53 patients (14 men, 39 women; mean age 71.6 years, range: $47-87$ years). Bilateral surgery was performed in 41 patients and unilateral in 12. Group A comprised 76 eyelids, among which 70 eyelids were assigned to group A1 and 6 to group A2. Group B comprised 18 eyelids. Thus, a fatty deposit was confirmed in the LPS \pm the levator aponeurosis in 88 eyelids (group $\mathrm{A} 1+$ group $\mathrm{B}=93.6 \%$ ). The remaining six eyelids (group A2, 6.4\%) had no apparent fatty deposit in the levator aponeurosis/LPS muscle.

There were no significant differences among the groups regarding the surgical side, phenylephrine test results, preoperative MRD-1, or levator function (all $p>0.050$ ). The percentage of eyelids with a double eyelid crease, high eyelid crease, or dehiscence or disinsertion of the levator aponeurosis did not differ among the groups (all $p>0.050$ ).

The non-fatty-deposit length was significantly greater in group A1 $(4.91 \pm 1.08)$ than in group B $(3.85 \pm 0.72)(p=$ $0.035)$. The levator aponeurosis was less advanced in groups A1 and A2 than in group B (both, $p<0.001$ ), although the eyelids in groups $\mathrm{A} 1$ and $\mathrm{A} 2$ obtained a higher MRD-1 than those in group B (both, $p<0.001$ ).

The logistic regression analysis (Table 2) showed that the non-fatty-deposit length $(p=0.004)$, response to phenylephrine eye drops $(p=0.008)$ and high eyelid crease $(p=$ 0.042 ) significantly influenced the surgical success. The presence or absence of dehiscence or disinsertion of the levator aponeurosis, the degree of levator function, or preoperative MRD-1 did not significantly influence the surgical success $(p>0.050)$.

There were no serious complications (e.g., infections, excess bleeding, subcutaneous haematoma and lagophthalmos) during the measurement period.

\section{Discussion}

The present study showed that the non-fatty-deposit length was greater in group A1 than in group B. In addition, the levator aponeurosis was more advanced in group B than in either group $\mathrm{A} 1$ or $\mathrm{A} 2$, whereas the postoperative MRD-1 was lower in group B. These results indicate that finding of a fatty deposit in the levator aponeurosis/LPS muscle intraoperatively and intraoperative measurement of the nonfatty-deposit length are useful predictors of surgical success following transcutaneous aponeurotic repair. Eyelids with a short non-fatty-deposit length may require a large amount of levator advancement or another surgical procedure, including combined Müller muscle resection or intraoperative conversion to suspension surgery. 


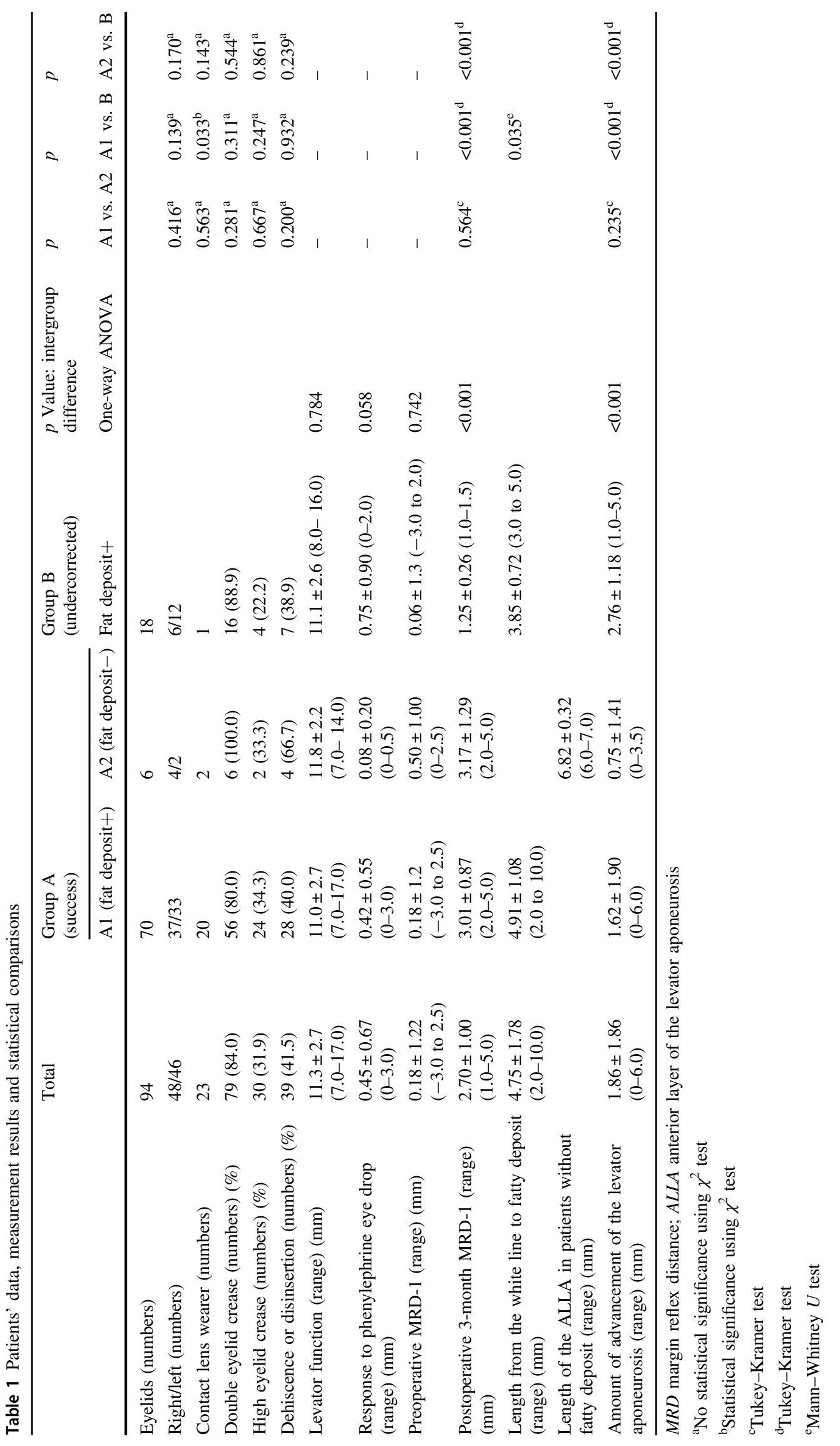


Table 2 Results of the logistic regression analysis

\begin{tabular}{ll}
\hline Variables & $p$ Value \\
\hline High eyelid crease & 0.042 \\
Dehiscence or disinsertion & 0.325 \\
Levator function & 0.740 \\
Response to phenylephrine eye drops & 0.008 \\
Preoperative MRD-1 & 0.480 \\
Non-fatty-deposit length & 0.004 \\
\hline
\end{tabular}

$M R D$ margin reflex distance

The incidence of fatty degeneration in the levator aponeurosis/LPS muscle is quite different among races $[3,13]$. Previous studies demonstrated fatty degeneration in almost all East Asian patients versus in only $25.6 \%$ of Caucasian patients [3, 13]. Most of the eyelids $(93.6 \%)$ in the present study showed fatty deposits in the levator aponeurosis/LPS muscle, similar to the results of a previous study of East Asian patients [3]. Unlike our macroscopic study, when fatty deposits can be detected more accurately using a microscopic examination [11], the true incidence of fatty deposit in the East Asian populations may be even higher. Invisible fatty deposits, however, may have little or negligible influence clinically.

Levator function is one of the key factors for determining the severity of blepharoptosis and selecting appropriate management [1]. Also, it is possibly affected by the amount of fatty deposit in the levator aponeurosis/LPS muscle because this deposit reduces the tractional force to the upper eyelid. No intergroup difference in levator function was shown in the present study, however, although postoperative MRD-1 was greater in groups A1 and A2 than in group B. These results were consistent with those in a previous study [13]. Although the study showed poor surgical outcomes and a revision rate that was higher for patients with fatty-appearing LPS muscle than for patients with normal-appearing muscle, there was no intergroup difference in the preoperative MRD-1 or levator function. The criteria for patient inclusion might explain this discrepancy. We excluded patients with poor levator function, so most patients had good levator function. In addition, a previous study included only patients with levator function of $\geq 10 \mathrm{~mm}$. These narrow ranges of levator function may prevent the comparative results from reaching statistical significance.

Variations in the vertical length of the levator aponeurosis ranging from 5 to $12 \mathrm{~mm}$ have been reported [14, 15]. These previous studies used the upper edge of the tarsal plate as a measurement reference point, but dehiscence or disinsertion of the levator aponeurosis from the tarsal plate may cause a measurement error [7], which could be responsible for the variation. In this study, we used the white line as a measurement reference point. Among the six eyelids with no fatty deposit, the standard deviation for the length of the ALLA was small $(0.32 \mathrm{~mm})$, indicating that the white line was a more reliable reference point for measuring the vertical length in relation to the ALLA.

In a previous study, the junction between the levator aponeurosis and LPS muscle was used as a reference point for advancing the levator aponeurosis [16]. As fatty degeneration extends from the LPS, however, it frequently occurs at the junction [2], resulting in some vagueness at that point. In contrast, the white line is away from the LPS, where fatty degeneration seldom occurs. In addition, eyelids with less fatty deposit, as in group $\mathrm{A} 1$, require less advancement of the levator aponeurosis. In such situations, the distance between the fixation point of the levator aponeurosis to the tarsal plate from the white line is shorter and more easily measured than that from the junction between the levator aponeurosis and LPS muscle.

We previously showed the occurrence of postoperative upper eyelid droop in patients with greater changes in the MRD-1 during the phenylephrine test [9]. This result was similar to that of the present study, showing that the degree of response to phenylephrine eye drops significantly influenced surgical success. In contrast, the intergroup difference in response to phenylephrine eye drops was small, without statistical significance, indicating that even a small difference in response could have a great effect on the postoperative MRD-1.

Patients with a high eyelid crease had significantly more favourable surgical results in the present study. The levator aponeurosis connects with the subcutaneous fascia through delicate interconnecting fibres [17]. The eyelids in East Asian individuals frequently have a downward extension of the preaponeurotic fat pad more inferiorly [6], which prevents formation of a high eyelid crease. Conversely, high eyelid creases may be found in less fatty eyelids, which could be more easily elevated. These factors may account for our results.

Our study is limited by several factors. This study had a retrospective design. Also, a relatively large number of patients were excluded. A larger number of included patients would provide more accurate results and greater statistical power. Another limitation was the inclusion of only Japanese patients. As the degree of fatty degeneration is variable among races [3, 13], the results of the present study may not be applicable to other nationalities. Our results were not available during transconjunctival ptosis repair because the fatty deposit is rarely examined intraoperatively. Although we evaluated the surgical outcomes at postoperative 3-month according to a previous study [18], the lack of long-term outcomes is a limitation of this study.

In conclusion, the length from the distal end of the ALLA to the most distal point of the fatty deposit was 
greater in group A1 than in group B. In addition, the levator aponeurosis was more advanced in group B than in either group $\mathrm{A} 1$ or $\mathrm{A} 2$, although the postoperative MRD-1 was lower in group B. These results imply that an intraoperative finding of a fatty deposit and intraoperative measurement of the non-fatty-deposit length could serve as useful predictors of surgical success after transcutaneous aponeurotic repair.

\section{Summary}

\section{What was known before}

- Fat deposition in the levator aponeurosis/levator palpebrae superioris muscle is a cause of aponeurotic blepharoptosis.

- A fatty deposit in the aponeurosis/LPS muscle is more prevalent in East Asians than in Caucasians, suggesting that fat degeneration has a greater impact on the clinical aspects of aponeurotic blepharoptosis in East Asian patients.

\section{What this study adds}

- Eyelids with less fatty deposits in the levator aponeurosis/levator palpebrae superioris muscle required less advancement of the levator aponeurosis and achieved more favourable surgical results.

- Intraoperative finding of a fatty deposit and intraoperative measurement of the non-fatty-deposit length may be useful predictive parameters for surgical success after transcutaneous aponeurotic repair.

Acknowledgements We thank Nancy Schatken, BS, MT (ASCP), from Edanz Group (www.edanzediting.com/ac) for editing a draft of this manuscript.

\section{Compliance with ethical standards}

Conflict of interest The authors declare that they have no conflict of interest.

\section{References}

1. Malhotra R, Salam A, Then SY, Grieve AP. Visible iris sign as a predictor of problems during and following anterior approach ptosis surgery. Eye. 2011;25:185-91.

2. Shore JW, McCord CD Jr. Anatomic changes in involutional blepharoptosis. Am J Ophthalmol. 1984;98:21-27.

3. Kim CY, Lee SY. Distinct features in Koreans with involutional blepharoptosis. Plast Reconstr Surg. 2015;135:1693-9.

4. Kakizaki H, Zako M, Nakano T, Asamoto K, Miyaishi O. The levator aponeurosis consists of two layers that include smooth muscle. Ophthal Plast Reconstr Surg. 2005;21:281-4.

5. Kakizaki H, Malhotra R, Selva D. Upper eyelid anatomy: an update. Ann Plast Surg. 2009;63:336-43.

6. Kakizaki H, Leibovitch I, Selva D, Asamoto K, Nakano T. Orbital septum attachment on the levator aponeurosis in Asians: in vivo and cadaver study. Ophthalmology. 2009;116:2031-5.

7. Sagili S. Anterior approach white-line advancement: a hybrid technique for ptosis correction. Ophthal Plast Reconstr Surg. 2015;31:478-81.

8. Boboridis K, Assi A, Indar A. Repeatability and reproducibility of upper eyelid measurements. Br J Ophthalmol. 2001;85:99-101.

9. Matsuda H, Shiba T, Takahashi Y, Tsuneoka H. Relationship between the phenylephrine test and eyelid droop after aponeurotic repair with the use of an epinephrine-containing local anaesthetic. Eye. 2018;32:93-8.

10. Park DH, Choi WS, Yoon SH, Song CH. Anthropometry of asian eyelids by age. Plast Reconstr Surg. 2008;121:1405-13.

11. Cahill KV, Buerger GF Jr, Johnson BL. Ptosis associated with fatty infiltration of Müller's muscle and levator muscle. Ophthal Plast Reconstr Surg. 1986;2:213-7.

12. Matsuda H, Shiba T, Takahashi Y, Tsuneoka H. Transcutaneous aponeurotic repair with small detachment of the levator aponeurosis for aponeurotic blepharoptosis in Japanese patients. J Plast Reconstr Aesthet Surg. 2018;71:425-30.

13. Tucker SM, Verhulst SJ. Stabilization of eyelid height after aponeurotic ptosis repair. Ophthalmology. 1999;106:517-22.

14. Kuwabara T, Cogan DG, Johnson CC. Structure of the muscles of the upper eyelid. Arch Ophthalmol. 1975;93:1189-97.

15. Anderson RL, Beard C. The levator aponeurosis. Attachments and their clinical significance. Arch Ophthalmol. 1977;95:1437-41.

16. McCord CD, Seify H, Codner MA. Transblepharoplasty ptosis repair: three-step technique. Plast Reconstr Surg. 2007; 120:1037-44.

17. Dutton JJ. The eyelid s and anterior orbit. In: Dutton JJ, editors. Atlas of Clinical and Surgical Orbital Anatomy. vol. 2 Philadelphia: Saunders; 2011. p. 129-64.

18. Linberg JV, Vasquez RJ, Chao GM. Aponeurotic ptosis repair under local anesthesia: prediction of results from operative lid height. Ophthalmology. 1988;95:1046-52. 